\title{
Aseptic meningitis after vaccination of the BNT162b2 mRNA COVID-19 vaccine
}

\author{
Kazuyuki Saito ${ }^{1}\left[\right.$ Taro Shimizu $^{2} \cdot$ Katsue Suzuki-Inoue $^{3} \cdot$ Tatsuhiro Ishida $^{2} \cdot$ Yoshiaki Wada $^{1}$
}

Received: 28 April 2021 / Accepted: 1 August 2021 / Published online: 11 August 2021

(c) Fondazione Società Italiana di Neurologia 2021

Dear editor,

Severe acute respiratory syndrome coronavirus 2 (SARSCoV2) infection has spread worldwide. The most promising strategy for successfully overcoming this coronavirus disease 2019 (COVID-19) pandemic is vaccination. Vaccination of the BNT162b2 messenger RNA (mRNA) against COVID-19 funded by Pfizer-BioNTech has recently started in Japan. The efficacy (94.8\% against COVID-19) and safety are proven [1]. Although uncommon, serious adverse events have been reported. Herein, we report a first case of aseptic meningitis after the first dose of intramuscular injection of the BNT162b2 mRNA COVID-19 vaccine.

A 42-year-old Japanese female nurse had a history of migraine, but she has had no obvious attack at least 9 months. She was just taking non-steroidal anti-inflammatory drugs (NSAIDs) and lomerizine hydrochloride orally as prophylaxis at that time. She has no history of allergy for food or cosmetics. Due to her professional history of contacting with patients who were treated with COVID-19, she had done SARS-CoV2 polymerase chain reaction (PCR) tests of a nasopharyngeal swab several times but never been positive before vaccination of the BNT162b2 mRNA vaccine. She received the first dose of the BNT162b2 mRNA vaccine $(30 \mu \mathrm{g})$ at our hospital 1 week before the appearance of severe headache and high fever $\left(38{ }^{\circ} \mathrm{C}\right)$. Although she took oral loxoprofen sodium hydrate as NSAIDs and acetaminophen because she thought that she had a migrane attack. Her headache

\section{Kazuyuki Saito \\ kaz_saito228@yahoo.co.jp}

1 Department of Neurology, Nissan Tamagawa Hospital, Seta 4-8-1, Setagaya, Tokyo 158-0095, Japan

2 Department of Pharmacokinetics and Biopharmaceutics, Institute of Biomedical Sciences, Tokushima University, Tokushima, Japan

3 Department of Clinical and Laboratory Medicine, Faculty of Medicine, Yamanashi University, Yamanashi, Japan getting worse, she injected sumatriptan succinate subcutaneously a day before the visit. In spite of the treatment, her headache was continued. Finally, she visited our hospital next day. On arrival, neck stiffness, Kernig sign, Brudzinski sign, eye ball tenderness, and other neurological signs were negative but only jolt accentuation was positive with headache and nausea. Her white blood cell count was 7,600/ $\mu \mathrm{L}$ (normal range 3,100-8,800/ $\mu \mathrm{L}$; neutrophils $69.2 \%$, eosinophils $1.7 \%$, basophils $0.3 \%$, lymphocytes $22.6 \%$, monocytes $6.2 \%$ ), although C-reactive protein level (CRP) was increased $(9.85 \mathrm{mg} /$ $\mathrm{dL}$; normal range $0-0.3 \mathrm{mg} / \mathrm{dL}$ ). Computed tomography (CT) of chest showed no sign of pneumonia. A cerebrospinal fluids (CSF) analysis revealed pleocytosis (528/3 $\mathrm{mm}^{3}$; polynuclear cells $34.8 \%$, mononuclear cells $64.1 \%$, eosinophils $1.1 \%$; Fig. 1), normal IgG index (0.54; normal range $0-0.73)$, and normal protein level $(35.7 \mathrm{mg} / \mathrm{dL}$; normal range $0-45.0 \mathrm{mg} / \mathrm{dL}$ ) with high opening pressure $\left(22 \mathrm{cmH}_{2} \mathrm{O}\right)$. SARS-CoV2 PCR tests of a nasopharyngeal swab and CSF were both negative. We evaluated the presence of spike-specific ( $\mathrm{Sp}$ ) SARS-CoV2 IgG and IgM; she was slightly positive for the Sp SARS-CoV2 IgG (393.4 AU/mL; cutoff 0-50 AU/mL; (Chemiluminescent Enzyme Immunoassay; Architect Quant IgG II, Abbott)), but she was qualitatively negative for the Sp SARS-CoV2 IgM. Brain magnetic resonance imaging showed normal findings, including contrast enhancement. She was diagnosed with aseptic meningitis. Blood and CSF cultures were negative. Her CSF Sp SARS-CoV2 IgM was negative, although her CSF Sp SARS-CoV2 IgG was detected (17.1 $\mathrm{AU} / \mathrm{mL}$ ). Acyclovir ( $3 \mathrm{mg} / \mathrm{kg} /$ day) was administered for several days until other viral antibody titers were negative. Her headache showed no improvement. We therefore treated her with 5 days intravenous methylprednisolone (500 mg/day) after 3 days from admission. After 5 days from admission, her headache and nausea were significantly improved. Serum CRP level was rapidly decreased $(0.36 \mathrm{mg} / \mathrm{dL})$ and a pleocytosis $\left(104 / 3 \mathrm{~mm}^{3}\right)$ and protein 


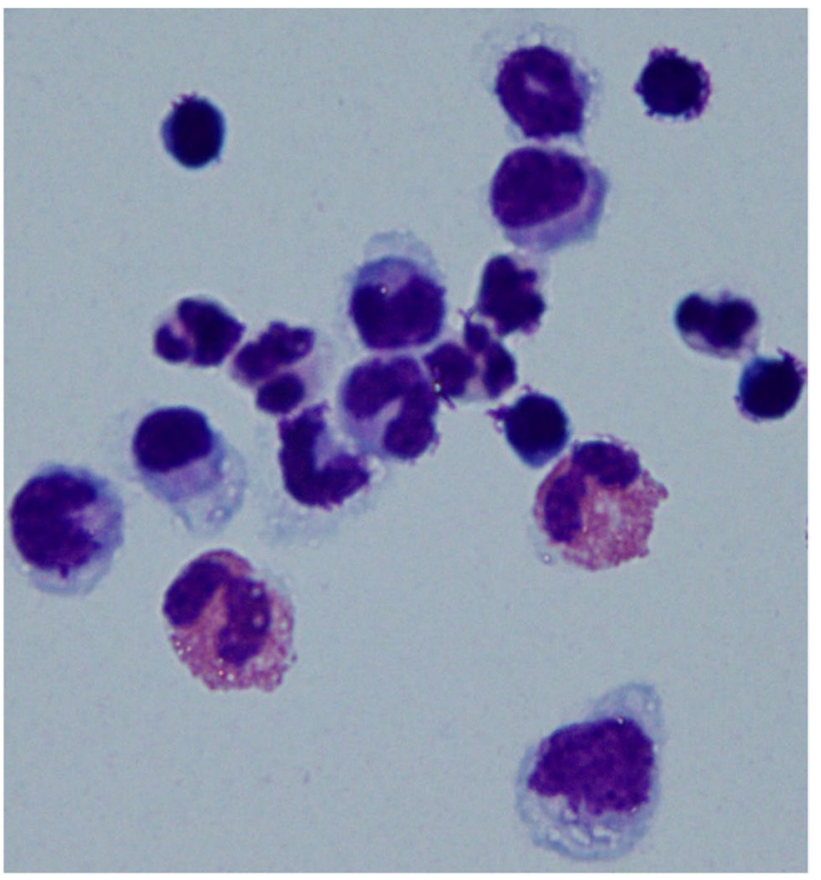

Fig. 1 Cytology of the cerebrospinal fluids at first lumbar puncture on the day of admission revealed eosinophils, polynuclear cells, and lymphocytes. May-Giemsa staining, $\times 100$

level $(21.0 \mathrm{mg} / \mathrm{dL})$ in CSF were getting improved on the same day. These results suggested the involvement of immune-mediated mechanism for the aseptic meningitis in the present case. She was discharged at 7 days from admission. She has never noticed headache, nausea, and fever at least 4 months after discharge. SARS-CoV2 PCR tests of a nasopharyngeal swab were all negative during the course of the disease.

We examined the antibodies against $\mathrm{S} 1$ domain of the Sp for SARS-CoV2, a characteristic structural component of the viral envelope, according to the previous report [2]. Although the S1 Sp SARS-CoV2 IgM antibodies were all negative, the S1 Sp SARS-CoV2 IgG antibodies were all increased, at days 7, 11, and 34 after receiving the first dose of the BNT162b2 mRNA COVID-19 vaccine. The vaccination by the BNT162b2 mRNA COVID-19 vaccine was effective for her acquired immunity for SARS-CoV2. As is already known, the BNT162b2 mRNA vaccine uses a lipid nanoparticle-based carrier system for stabilization by polyethylene glycol (PEG) [3], which has never been used for an approved vaccine and believed to be an antigen for anaphylaxis after receiving the BNT162b2 mRNA COVID-19 vaccine [3]. According to the previous reports [4], we also checked the anti-polyethylene glycol (PEG) IgG and IgM antibodies of acute and recovery phase at days 7,11 , and 34 in her sera which were all negative. The factors other than PEG need to be considered.
The appearance of aseptic meningitis after vaccinations against mumps, measles and rubella and influenza has been reported. However, the cause of meningitis after vaccination is exactly unclear. There was a report of brain tissues from 13 autopsies of people who died of COVID19. Serial section analyses of the COVID-19 brain showed that the endothelial cells of the microvessels contained the spike glycoprotein. The $\mathrm{S} 1$ spike subunit was localized to the endothelia of microvessels in the mice brain and showed co-localization with TNF $\alpha$, IL-6, ACE2 and C5-9. It was shown that the full-length $\mathrm{S} 1$ subunit of the spike protein of SARS-CoV2 alone is capable of inducing systemic microendothelial cell damage in mice with a cognate pattern of complement activation and increased cytokine expression [5]. There is a possibility that the molecular mimicry elicited by the protein, which was produced by vaccination, could cause an autoimmune meningitis. The autoimmune response or $\mathrm{S} 1$ protein produced by the vaccination itself may cause the disruption of blood-brain barrier which induced aseptic meningitis in the present case.

This is the first report of aseptic meningitis after administration of the BNT162b2 mRNA vaccine against COVID-19. We consider the vaccine to be effective; however, the present case is thought provoking.

Acknowledgements The authors would like to thank Shinji Ogihara, Department of Clinical and Laboratory Medicine, Faculty of Medicine, University of Yamanashi, for testing SARS-CoV2 PCR in the CSF, the Pharmacy (Mizuho Enoki, Kazuyo Takeba and Seiji Tagawa), the Inspection unit (Kazumasa Koga, Masami Kawahara, Mihoko Inaki, Junpei Hirai, Ritsu Maejima, Leo Takahashi and Jun Kanno), the Infection control team (Naoko Yokomizo, Ayaka Takano, Sumiko Hasegawa, Shun Tanaka and Chiemi Takemoto) and Saori Fujii and all of the Nursing team for the contribution to the team of COVID-19 and Masaki Kobayashi, Department of Neurology, Nissan Tamagawa Hospital and Toyokazu Saito, Emeritus professor, Kitasato University for helpful advice of the manuscript.

\section{Declarations}

Ethical approval None.

Informed consent Informed consent was obtained from the patient.

Conflict of interest The authors declare no competing interests.

\section{References}

1. Polack FP, Thomas SJ, Kitchin N et al (2020) Safety and efficacy of the BNT162b2 mRNA Covid-19 vaccine. N Eng J Med 383:2603-2615

2. Kim E, Erdos G, Huang S et al (2020) Microneedle array delivered recombinant coronavirus vaccines: immunogenicity and rapid translational development. EBioMedicine 55:1-12 
3. Castells MC, Phillips EJ (2021) Maintaining safety with SARSCoV2 vaccines. N Eng J Med 384:643-649

4. Oh Y, Niijima H, Kawahara Y et al (2020) An immediate hypersensitivity reaction induced by PEGylated recombinant factor VIII. Haemophilia 26:e236-e239
5. Nuovo GJ, Magro C, Shaffer T et al (2020) Ann Diagn Pathol 51:151682. https://doi.org/10.1016/j.anndiagpath.2020.151682

Publisher's note Springer Nature remains neutral with regard to jurisdictional claims in published maps and institutional affiliations. 